\title{
Advanced computational and statistical multiparametric analysis of Susceptibility- Weighted Imaging to characterize gliomas and brain metastases.
}

\section{Antonio Di leva, ${ }^{1,2,3}$ Carlo Russo, ${ }^{1,2}$ Pierre-Jean Le Reste, ${ }^{4}$ John S. Magnussen, ${ }^{1,5}$ Gillian Heller ${ }^{6}$}

${ }^{1}$ Computational NeuroSurgery (CNS) Lab, Macquarie University, Sydney, NSW, Australia

${ }^{2}$ Department of Clinical Medicine, Faculty of Medicine, Health and Human Sciences, Macquarie University, Sydney, NSW, Australia

${ }^{3}$ Macquarie Neurosurgery, Sydney, Australia

${ }^{4}$ Department of Neurosurgery, University Hospital, Rennes, France

${ }^{5}$ Macquarie Medical Imaging, Macquarie University, Sydney, New South Wales, Australia

${ }^{6}$ Department of Statistics, Macquarie University, Sydney, NSW, Australia

\section{Corresponding Author}

Prof. Antonio Di leva, MD, PhD, FRACS

Macquarie Neurosurgery

Level 2, Suite 201, 2 Technology Place

Macquarie University

NSW 2109 - Australia

Email: antonio.diieva@mq.edu.au 
Abstract. Susceptibility-weighted imaging (SWI) is a technique useful for evaluation of the internal structures of brain tumors, including microvasculature and microbleeds. Intratumoral patterns of magnetic susceptibility can be quantified by means of fractal analysis. Here, we propose a radiomics methodological pipeline to merge advanced fractal-based computational modelling with statistical analysis to objectively characterize the fingerprint of gliomas and brain metastases. Forty-seven patients with glioma (grades II-IV, according to the WHO 2016 classification system) and fourteen with brain metastases underwent 3 Tesla MRI using a SWI protocol. All images underwent computational analysis aimed to quantify three Euclidean parameters (related to tumor and SWI volume) and five fractal-based parameters (related to the pixel distribution and geometrical complexity of the SWI patterns). Principal components analysis, linear and quadratic discriminant analysis, K-nearest neighbor and support vector machine methods were used to discriminate between tumor types. The combination of parameters offered an objective evaluation of the SWI pattern in gliomas and brain metastases. The model accurately predicted $88 \%$ of glioblastoma, according to the quantification of intratumoral SWI features, failing to discriminate the other types. SWI is not normally used to classify brain tumors, however fractal-based multi-parametric computational analysis can be used to characterize intratumoral SWI patterns to objectively quantify tumors-related features. Specific parameters still have to be identified to provide completely automatic computerized differential diagnosis.

Keywords Fractal analysis - Brain Tumor - Radiomics - SWI - Susceptibility-Weighted Imaging Principal Component Analysis - Support Vector Machine

\author{
Abbreviations \\ FD Fractal Dimension \\ WHO World Health Organization \\ GBM Glioblastoma \\ ROI Region of interest \\ SWI Susceptibility-weighted imaging
}

The preliminary results of this study were presented at the $15^{\text {th }}$ Annual World Congress of the Society for Brain Mapping \& Therapeutics (SBMT), 13 April 2018, Los Angeles, California, USA 


\section{Introduction}

With the introductions of more complex and potentially more sensitive imaging techniques in neuroradiology, there is a growing need for quantitative analysis to provide objective measures of radiological features in conjunction with the qualitative description by the specialist radiologist. Artificial intelligence techniques are being rapidly deployed in neuroradiology and radiomics [1, 2], many of which may benefit from new image markers to describe and characterize regions of interest (ROI), quite apart from new computerized parameters which could quantify specific features in an objective way.

Susceptibility-weighted imaging (SWI) is an imaging technique useful for the evaluation of the internal structures of brain tumors, including microvasculature and microbleeds $[3,4]$. SWI can demonstrate intra-tumor heterogeneity; that is, the intralesional signal patterns and edge irregularity[5].

In the field of quantitative neuroradiology and clinical neurosciences, fractal-based computational modelling has been introduced for the characterization of intrinsic features (e.g., microvascular patterns) [6-8]. The intratumoral patterns of magnetic susceptibility can be quantified by means of fractal analysis [9]. In prior research, we have demonstrated that the fractal dimension of intratumoral SWI patterns, as seen at ultra-high-field (7 Tesla) [5] and high-field (3 Tesla) [10], is a valid morphometric image marker to estimate glial brain tumor grade, which is useful in cancer and neuroimaging research as well as in the clinical setting.

Here, we propose a methodological pipeline merging advanced fractal-based computational modelling with statistical analysis to objectively characterize the fingerprint of gliomas and brain metastases. The current model aims to generate neuroimaging fingerprints of brain tumors, to help neuroradiologists and clinicians to characterize and follow-up patients undergoing serial radiological investigations.

\section{$2 \quad$ Materials and methods}

2.1 Patients. Following informed consent and local ethics committee approval, we retrospectively analyzed the pre-surgical imaging of 61 patients (mean age 51 +/- 16 years; range 2477) who underwent multimodal imaging, including SWI. The pre-operative images were compared with the post-surgical pathological analysis of the sample, consisting of: 13 grade II gliomas ( 9 oligodendroglioma, 1 oligoastrocytoma, 3 astrocytoma); 10 grade III gliomas (7 anaplastic oligodendrogliomas, 3 anaplastic astrocytomas), and 24 grade IV gliomas (glioblastoma); 14 metastases ( 3 melanomas, 3 mammary carcinoma, 6 pulmonary adenocarcinomas, 1 gallbladder carcinoma, and 1 uterine carcinoma). 
2.2 MR Imaging. All MRIs were performed on a 3-T Philips Achieva scanner (Philips, Amsterdam, the Netherlands). The sequences used in this study included T1-weighted isotropic gradient-echo imaging before and after gadolinium infusion, $\mathrm{T} 2$ fluid-attenuated inversion recovery imaging, and 2 types of 3-dimensional SWI depending on the year of acquisition (matrix $=512 \times 512$, resolution $=0.43 \times 0.43 \times 0.5 \mathrm{~mm}, 200$ slices, repetition time $/$ echo time $=22 / 32 \mathrm{~ms}$ or matrix $=512 \mathrm{x}$ 512 , resolution $=0.43 \times 0.43 \times 1.2 \mathrm{~mm}, 84$ slices, repetition time $/$ echo time $=28.3 / 38.3 \mathrm{~ms}$ ). The tumors were contoured by consensus on each slice of native susceptibility-weighted images by a neurosurgeon and a neuroradiologist who were blinded to the histology results. The segmentation was produced with the help of all sequences rigidly registered on SWI using a 12-degree of freedom algorithm (MIPAV - Medical Image Processing, Analysis, and Visualization, which is freely distributed at http://mipav.cit.nih.gov/).

2.3 Postprocessing image analysis. All the images were filtered by using the Brightness Progressive Normalization algorithm (http://www.fractal-lab.org/Downloads/bpn_algorithm.html), designed to homogenize the grayscale level of the SWI signals across the dataset [9]. Following segmentation, each region of interest (ROI) represented the tumor volume. Three Euclidean parameters were calculated from the ROI: volume (defined as P5, see Table 1); the threshold volume (defined as the volume of the structure obtained by intersecting the ROI and the gray level threshold) (P6); and the ratio between the threshold volume and the ROI volume (P7).

\begin{tabular}{|l|l|l|}
\hline P1 & 3D Histogram FD & Complexity of the gray level distribution of the ROI \\
\hline P2 & 3D FD & General complexity of the shape of the ROI \\
\hline P3 & 3D inner FD & $\begin{array}{l}\text { FD of the SWI pattern in relation to the perimeter/area ratio of each } \\
\text { grey level }\end{array}$ \\
\hline P4 & SWI 3D FD & $\begin{array}{l}\text { Complexity of the pattern related to the 3D shape of the gray level } \\
\text { threshold into the ROI }\end{array}$ \\
\hline P5 & ROI volume $\mathbf{( c m}^{\mathbf{3}}$ ) & Tumor Volume \\
\hline P6 & Threshold Volume & $\begin{array}{l}\text { Image volume obtained by intersecting the ROI and the gray level } \\
\text { threshold }\end{array}$ \\
\hline P7 & Vol/ROI Vol ratio & P6/P5 ratio \\
\hline P8 & 3D internal FD Max & Like P3, but evaluating only the maximum values \\
\hline
\end{tabular}

Table 1 Definitions of the computed Euclidean (P5-P7) and fractal-based (P1-P4 and P8) parameters. FD: fractal dimension.

2.4 Fractal analysis. The box counting method was applied to assess the fractal dimension (FD). We used custom software developed in $\mathrm{C}++$ for image and fractal analysis (http://www.fractallab.org/Downloads/FDEstimator.html). After image normalization and binarization, 5 fractal parameters were evaluated (Fig. 1), based on the FD, which is a non-integer number between 0 and 3 quantifying the geometric complexity of the analyzed object [11, 12]: 
1) the 3D Histogram fractal dimension (P1), defined as the FD computed on the histogram of gray values in the selected ROI, evaluating gray level distribution rather than pixel arrangement. The value is obtained considering the distribution of the frequencies of each gray level of the pixels in the selected volume. The complexity of this distribution is given by the FD obtained evaluating the means of subsets of the distribution on different scales. This parameter evaluates gray level distribution but not the arrangement of the pixels, i.e. the same pixels with a different arrangement will have the same 3D Histogram FD.

2) the 3D FD (P2), defined as the FD of the 3D surface of the ROI shape. The ROI selected on each image forms a 3D object, the complexity of which is variable. This complexity is evaluated by means of the 3D box counting algorithm [11]. This parameter is able to evaluate complexity of the ROI shape but does not consider the pixel information of the images.

3) the 3D inner FD (P3), defined as the FD of the 3D internal grey levels distribution, obtained by the perimeter/area ratio of each contour line obtained sliding the gray level threshold; each grey level generates a shape, and the complexity of the non-linear perimeter/area ratio graph is evaluated by its fractal dimension. In this way all the useful information of the gray level values of the pixels and their distribution are considered in the FD evaluation. It is a more sensitive parameter compared to the SWI 3D FD (see below) and the 3D Histogram FD. This parameter is related to the change of the pixel gray value, its position, or both. It considers a more general rather than local complexity.

4) the SWI 3D FD (P4), defined as the FD of the 3D surface of the selected structure obtained by intersecting the ROI with a gray level threshold of the pixels, i.e., pixels that have the gray level included into a selected threshold of gray values and are inside the selected ROI. This parameter evaluates the general complexity of the SWI patterns. The segmentation of the SWI pattern forms a 3D object with a more or less complex 3D shape. The measure of the complexity of this shape is obtained by evaluating its FD using the 3D box counting algorithm. This parameter is able to evaluate the complexity of the shape but does not change when the internal gray levels of the pixel are different, when all the gray levels remains included into the selected threshold.

5) the 3D internal FD max (P8), defined as the 3D internal FD (like P3), but evaluating the FD on a loglog plot having only values up to the maximum extension of the perimeter in the perimeter/area ratio, making it an estimator of the distribution of the gray level and pixel distribution. 

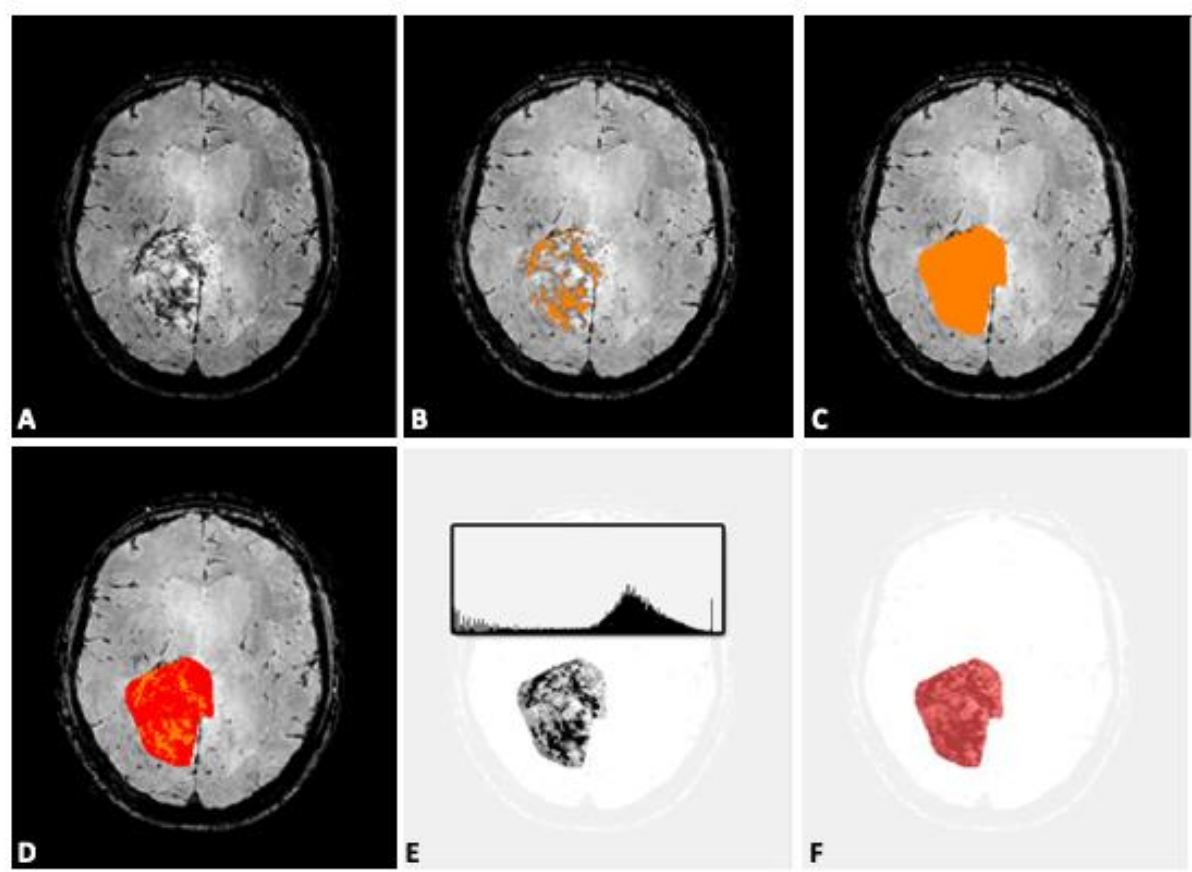

Fig. 1 A) SWI of a right-sided glioblastoma. B) Automatic segmentation of the intratumoral SWI pattern for computing of the 3D FD (P2), evaluating the complexity of the heterogeneity of the SWI signal. C) ROI volume (P5) on the entire slices' stack. D) Volume/ROI volume ratio. E) 3D Histogram FD (P1), evaluating the gray levels distribution; F) 3D inner FD (P3), evaluating the gray level and pixels' distribution.

2.5 Statistical analysis. The data was pre-processed using Box-Cox transformations to normality [13], in order to maximize discrimination between groups. Principal component analysis was performed on the transformed variables, and the principal components explaining most of the variation were carried forward to the discrimination procedures. Linear and quadratic discriminant analysis, K-nearest neighbor and support vector machine methods [14] were implemented in order to assess their ability to discriminate between types.

\section{$3 \quad$ Results}

A matrix of plots of the raw data is given in Fig. 2. 
bioRxiv preprint doi: https://doi.org/10.1101/2020.04.24.060830; this version posted April 25, 2020. The copyright holder for this preprint (which was not certified by peer review) is the author/funder, who has granted bioRxiv a license to display the preprint in perpetuity. It is made available under aCC-BY-NC-ND 4.0 International license.

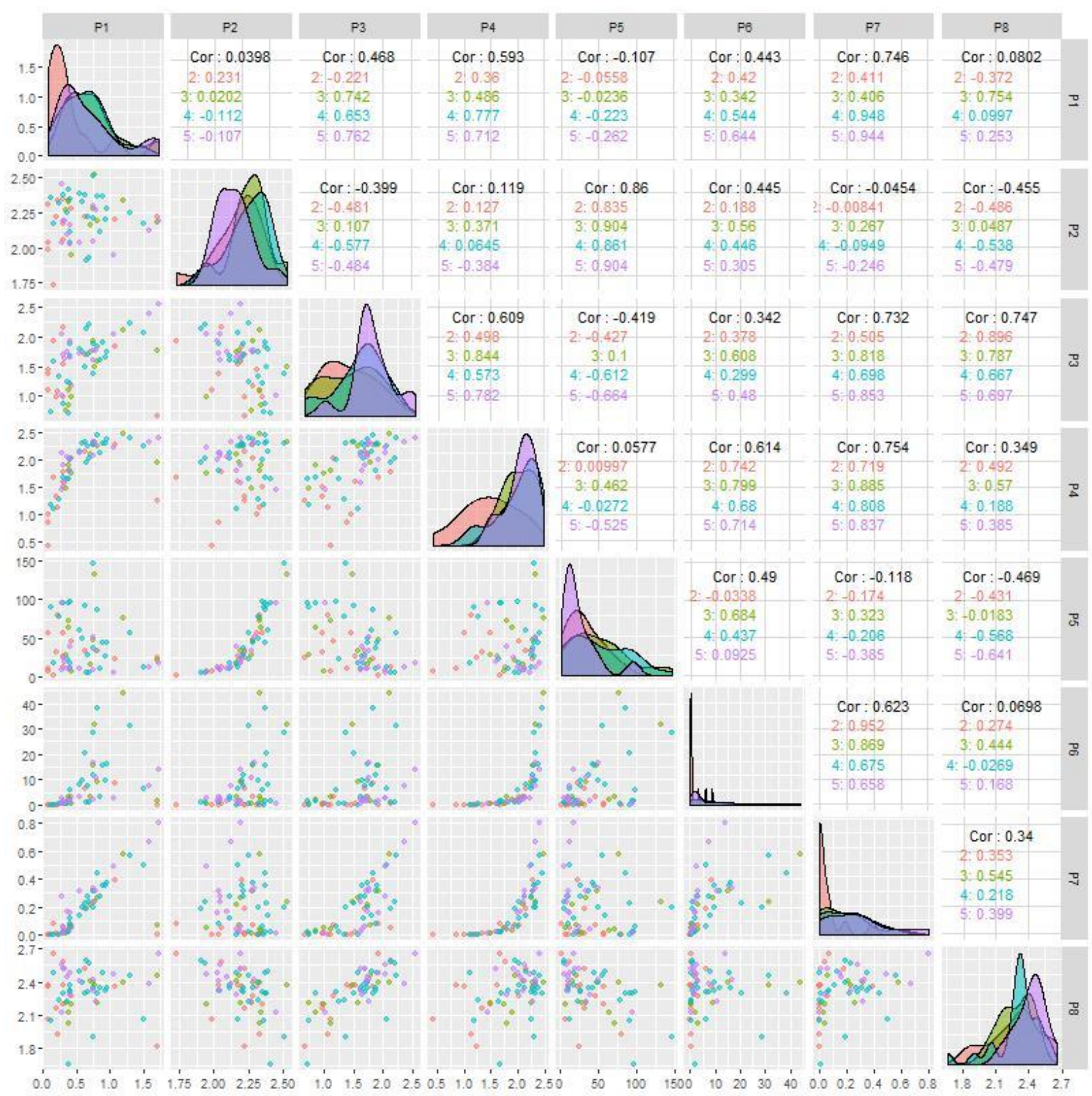

Figure 2 Matrix of plots of raw data. Density estimates by type are shown on the diagonal; bivariate scatterplots between pairs of variables below the diagonal; and Pearson's correlation coefficients above the diagonal. Glioma grade II = pink, grade III = green, Glioblastoma = blue, Metastasis = purple.

The distributions of several of the variables are severely skewed, and many of the bivariate scatterplots reveal nonlinear relationships. As pre-processing of the data to approximate normality and linearity is advantageous in analysis, we used Box-Cox analysis to find transformations $\mathrm{x}^{\beta}$ to achieve this. In all cases we rounded the Box-Cox estimate of the power $\beta$ to a reasonable number. The transformations shown in Table 2 were applied. 
bioRxiv preprint doi: https://doi.org/10.1101/2020.04.24.060830; this version posted April 25, 2020. The copyright holder for this preprint (which was not certified by peer review) is the author/funder, who has granted bioRxiv a license to display the preprint in perpetuity. It is made available under aCC-BY-NC-ND 4.0 International license.

Table 2 Power $\beta$ used in Box-Cox transformations.

\begin{tabular}{|l|l|}
\hline$\beta$ & Parameter(s) \\
\hline 0.25 & $5,6,7$ \\
\hline 0.5 & 1 \\
\hline 1.5 & 3 \\
\hline 2.5 & 4 \\
\hline 3.5 & 2 \\
\hline 4.5 & 8 \\
\hline
\end{tabular}

The plot matrix of the transformed variables is shown in Fig. $\mathbf{3 .}$

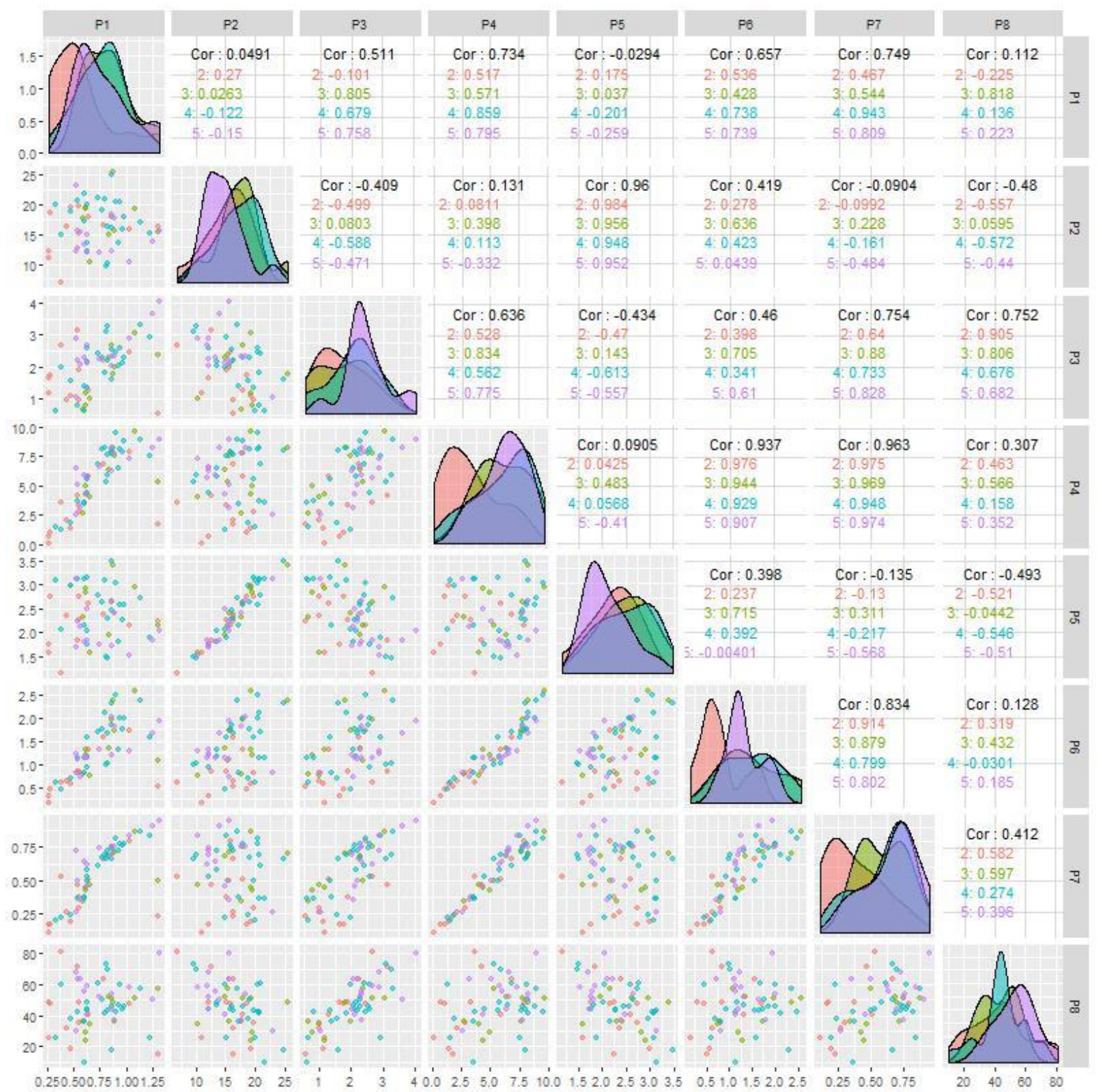

Figure 3 Matrix of plots of transformed data. Density estimates by type are shown on the diagonal; bivariate scatterplots between pairs of variables below the diagonal; and Pearson's correlation coefficients above the diagonal. Glioma grade $\mathrm{II}=$ pink, grade $\mathrm{III}=$ green, Glioblastoma = blue, Metastasis $=$ purple. 
The distributions are generally closer to normality, and relationships have been linearized in many cases (with a resultant increase in Pearson's correlation). Pairs of transformed variables exhibiting very high linear correlation (> 0.9 ) are $\mathrm{P} 2$ and $\mathrm{P} 5, \mathrm{P} 4$ and $\mathrm{P} 6$, and $\mathrm{P} 4$ and $\mathrm{P} 7$, showing high correlations between Euclidean parameters and fractal ones (above all, 3D FD and SWI 3D FD). P1-P4, P1-P6 and P3-P6 pairings had moderate correlations. The principal components analysis on the transformed variables gave the percent of variance explained as shown in Table 3. Three principal components explain $94.1 \%$ of the variance; four PCs explain $97.6 \%$.

\begin{tabular}{|l|l|l|}
\hline Principal component & Percent of variance & Cumulative percent \\
\hline 1 & 51.6 & 51.6 \\
\hline 2 & 33.9 & 85.5 \\
\hline 3 & 8.6 & 94.1 \\
\hline 4 & 3.5 & 97.6 \\
\hline 5 & 1.6 & 99.2 \\
\hline 6 & 0.5 & 99.7 \\
\hline 7 & 0.2 & 99.9 \\
\hline 8 & 0.1 & 100.0 \\
\hline
\end{tabular}

Table 3 Principal component analysis of transformed variables.

We investigated the use of the following discrimination methods on the PCs: linear discriminant analysis, quadratic discriminant analysis, K-nearest neighbors, and support vector machines (SVM), based on either three or four principal components. SVMs based on four principal components yielded the best results. The classification of the actual types is shown in Table 4. The overall percent of cases correctly classified by the method was $35 / 61=57 \%$. Glioblastoma had the best classification rate of $88 \%$ and grade III glioma the worst at $20 \%$.

\begin{tabular}{|c|c|c|c|c|c|c|}
\hline & & Predicted & type & & & \\
\hline Actual type & Glioma II & Glioma III & GBM & Metastasis & Total & Percent correctly classified \\
\hline Glioma II & 7 & 0 & 6 & 0 & 13 & $54 \%$ \\
\hline Glioma III & 1 & 2 & 7 & 0 & 10 & $20 \%$ \\
\hline Glioblastoma & 2 & 0 & 21 & 1 & 24 & $88 \%$ \\
\hline Metastasis & 0 & 0 & 9 & 5 & 14 & $36 \%$ \\
\hline
\end{tabular}

Table 4 Classification of actual types using support vector machine method on four principal components.

\section{Discussion}

SWI sequences provide information on venous vasculature, hemorrhage, iron deposits, and very small vascular structures within the central nervous system (including brain tumors) [4, 15-17]. Fractal geometry has been demonstrated to offer appropriate tools to quantify irregular-shaped biological objects, including microvascular and SWI patterns [8], that can be used in automated algorithms for counting microbleeds [18], quantitative susceptibility mapping [19], percentage-wise quantification of intratumoral-susceptibility signals [20], local image variance [21] and other quantitative 
methodologies [16]. The fractal dimension, the most used parameter in fractal geometry, has been shown as a reliable numerical index to objectively quantify geometrical complexity of microvascular patterns in brain tumors [22]. The fractal dimension is a characteristic of irregularly shaped structures that maintains a constant level of complexity over a limited range of scales $[8,23]$. Our previous findings showed that lower FD values of the intratumoral SWI patterns are associated with tumoral microvasculature, while higher values (more "space-filling" and geometrically complex) are associated with microbleeds and necrotic areas [5].

Although not discriminative, the computed parameters quantified in an objective way some intratumoral features, i.e., the SWI patterns of gliomas and brain metastases. This means that the specific fingerprint related to tumors can be quantified by means of radiomics fractal-based parameters. Although an overall percent of $57 \%$ cases correctly classified by the method appears to be low, it has to be emphasized that SWI is generally not used for grading and typing in the clinical setting of brain tumors neuroimaging. Nevertheless, SWI's capacity to visualize intratumoral microstructural features can be linked with quantitative tools, with the aim to characterize the neoplastic architecture and eventually follow it up over time. Advantages include: once the pipeline is standardized, the computed parameters are objective and independent from any operator-dependent variability; the methodology lacks the necessity to augment data when the dataset is small, as it is instead required in Al-based computation of small samples. Limitations are related to the fact that different protocols to acquire SWI features may create a variability in the inputs to be analyzed; moreover, the computation of the parameters is based on the box counting algorithm, which is a valid tool in mathematics and research but not well-known and widespread in the clinical setting. Moreover, the manual segmentation of the ROIs is time-consuming and can be affected by operatordependent bias. Once again, it is shown that grade III gliomas are the most difficult ones to be differentiate according to their neuroimaging fingerprinting, at least in terms of SWI patterns $[5,10]$. Despite such limitations, we believe that the fractal-based computational methodology can become a reliable tool in the neuroradiological armamentarium for the characterization of intrinsic features of brain tumors. Moreover, the present analysis has been applied to an MRI sequence, SWI, which is generally not used for typing and grading of brain tumours. Our results show a proof-of-concept of the applicability of the radiomics analysis to SWI. In future studies, complementary computational analyses will be applied to multimodal imaging for higher diagnostic predictivity.

\section{Conclusion}

A computational pipeline is proposed to provide a standardized diagnostic tool for the medical community. Future studies may include the combination of morphometric Euclidean and fractal parameters, merged with advance image analysis and statistical tools, as well as deep learning 
methodologies, to objectively characterize brain tumors, at benefit of automatic diagnosis and followup of regions of interest in neuroimaging.

\section{Acknowledgments}

Prof. Antonio Di leva received the 2019 John Mitchell Crouch Fellowship from the Royal Australasian College of Surgeons (RACS), which, along to Macquarie University co-funding, supported the Computational NeuroSurgery (CNS) Lab.

\section{Funding}

No funding was received for this study.

\section{Conflict of interest}

The authors declare that they have no conflict of interest.

\section{Ethical approval}

All procedures performed in the studies involving human participants were in accordance with the ethical standards of the institutional and/or national research committee and with the 1964 Helsinki Declaration and its later amendments or comparable ethical standards.

\section{Informed consent}

Informed consent was obtained from all individual participants included in the study. 


\section{References}

1. Senders JT, Arnaout O, Karhade A V, et al (2018) Natural and Artificial Intelligence in Neurosurgery: A Systematic Review. Neurosurgery 83:181-192 . doi: 10.1093/neuros/nyx384

2. Rudie JD, Rauschecker AM, Bryan RN, et al (2019) Emerging Applications of Artificial Intelligence in Neuro-Oncology. Radiology 290:607-618. doi: 10.1148/radiol.2018181928

3. Haacke EM, Mittal S, Wu Z, et al (2009) Susceptibility-weighted imaging: technical aspects and clinical applications, part 1. AJNR American J Neuroradiol 30:19-30 . doi: 10.3174/ajnr.A1400; 10.3174/ajnr.A1400

4. Di leva A, Lam T, Alcaide-Leon P, et al (2015) Magnetic resonance susceptibility weighted imaging in neurosurgery: current applications and future perspectives. J Neurosurg 123:1463-1475 . doi: 10.3171/2015.1.JNS142349

5. Di leva A, Göd S, Grabner G, et al (2013) Three-dimensional susceptibility-weighted imaging at 7 T using fractal-based quantitative analysis to grade gliomas. Neuroradiology 55:35-40 . doi: 10.1007/s00234-012-1081-1

6. Di leva A, Grizzi F, Jelinek H, et al (2014) Fractals in the neurosciences, part I: General principles and basic neurosciences. Neuroscientist 20:403-417 . doi: 10.1177/1073858413513927

7. Di leva A, Esteban FJ, Grizzi F, et al (2015) Fractals in the neurosciences, part II: Clinical applications and future perspectives. Neuroscientist 21:30-43 . doi: 10.1177/1073858413513928

8. Di leva A (2016) The Fractal Geometry of the Brain. Springer, New York

9. Di leva A, Matula C, Grizzi F, et al (2012) Fractal analysis of the susceptibility weighted imaging patterns in malignant brain tumors during antiangiogenic treatment: technical report on four cases serially imaged by $7 \mathrm{~T}$ magnetic resonance during a period of four weeks. World Neurosurg 77:785.e11-785.e21 . doi: 10.1016/j.wneu.2011.09.006

10. Di leva A, Le Reste P-J, Carsin-Nicol B, et al (2016) Diagnostic Value of Fractal Analysis for the Differentiation of Brain Tumors Using 3-Tesla Magnetic Resonance Susceptibility-Weighted Imaging. Neurosurgery 79:839-846 . doi: 10.1227/NEU.0000000000001308

11. Mandelbrot B (1967) How long is the coast of britain? Statistical self-similarity and fractional dimension. Science 156:636-638 . doi: 156/3775/636 [pii]

12. Mandelbrot BB (1982) The Fractal Geometry of Nature. W.H. Freeman, New York

13. Box, G.E.; Cox DR (1964) An analysis of transformations. J R Stat Soc Ser B 211-252

14. James, G.; Witten, D.; Hastie, T.; Tibshirani R (2013) An Introduction to Statistical Learning with Applications in R. Springer, New York

15. Rauscher A, Sedlacik J, Barth M, et al (2005) Magnetic susceptibility-weighted MR phase imaging of the human brain. AJNR Am J Neuroradiol 26:736-42

16. Hori M, Mori H, Aoki S, et al (2010) Three-dimensional susceptibility-weighted imaging at $3 \mathrm{~T}$ using various image analysis methods in the estimation of grading intracranial gliomas. Magn Reson Imaging 28:594-598 . doi: 10.1016/j.mri.2010.01.002; 10.1016/j.mri.2010.01.002 
bioRxiv preprint doi: https://doi.org/10.1101/2020.04.24.060830; this version posted April 25, 2020. The copyright holder for this preprint (which was not certified by peer review) is the author/funder, who has granted bioRxiv a license to display the preprint in perpetuity. It is made available under aCC-BY-NC-ND 4.0 International license.

17. Di leva A, Tschabitscher M, Galzio RJ, et al (2011) The veins of the nucleus dentatus: Anatomical and radiological findings. Neuroimage 54:74-79

18. Zou X, Hart BL, Mabray M, et al (2017) Automated algorithm for counting microbleeds in patients with familial cerebral cavernous malformations. Neuroradiology 59:685-690 . doi: 10.1007/s00234017-1845-8

19. Li X, Chen L, Kutten K, et al (2019) Multi-atlas tool for automated segmentation of brain gray matter nuclei and quantification of their magnetic susceptibility. Neuroimage 191:337-349. doi: 10.1016/j.neuroimage.2019.02.016

20. Radbruch A, Graf M, Kramp L, et al (2012) Differentiation of brain metastases by percentagewise quantification of intratumoral-susceptibility-signals at 3Tesla. Eur J Radiol 81:4064-4068. doi: 10.1016/j.ejrad.2012.06.016; 10.1016/j.ejrad.2012.06.016

21. Grabner G, Kiesel B, Wöhrer A, et al (2017) Local image variance of 7 Tesla SWI is a new technique for preoperative characterization of diffusely infiltrating gliomas: correlation with tumour grade and IDH1 mutational status. Eur Radiol 27:1556-1567 . doi: 10.1007/s00330-016-4451-y

22. Di leva A (2012) Fractal analysis of microvascular networks in malignant brain tumors. Clin Neuropathol 31:342-351

23. Losa GA (2009) The fractal geometry of life. Riv Biol 102:29-59 